\title{
Comparative study of conventional and hydromechanical deep drawing processes based on finite element analysis
}

\author{
Mohammed Abbadeni, Ibrahim Zidane, Hamou Zahloul, Zakaria Madaoui \\ University of Chlef, Rheology and Mechanics Laboratory, Algeria \\ mohamed.abbadeni@yahoo.fr, bttps://orcid.org/0000-0003-0681-9669 \\ zidane_bra@botmail.com \\ zabloulh@yahoo.fr, bttps://orcid.org/0000-0003-2760-2872 \\ zkrmadaoui@yahoo.fr,.https://orcid.org/0000-0002-6653-0544
}

\begin{abstract}
A two-dimensional finite element analysis has been performed to investigate hydromechanical and conventional deep drawing processes. The effects of the fluid structure interaction on the material formability, the strain and the thickness distributions are investigated. Large plastic deformation is observed in the plank at the bottom and the corner of the punch. Plastic deformation distribution results show a difference between the hydromechanical deep drawing process and the conventional deep drawing which is due to the friction effect. This latter represents one of the major features of the hydromechanical deep drawing process. Concerning the formability of the blank, the obtained results show that the hydromechanical deep drawing process gives a better formability than the conventional deep drawing process.
\end{abstract}

KEYwORDs. Hydromechanical deep drawing; Conventional deep drawing; Finite element analysis; Fluid pressure.

\section{OPEN ACCESS}

Citation: Abbadeni, M., Zidane, I., Zahloul, H., Madaoui, Z., Comparative study of conventional and hydromechanical deep drawing processes based on finite element analysis, Frattura ed Integrità Strutturale, 49 (2019) 282-290.

Received: 13.02 .2019

Accepted: 30.04 .2019

Published: 01.07.2019

Copyright: (C) 2019 This is an open access article under the terms of the CC-BY 4.0, which permits unrestricted use, distribution, and reproduction in any medium, provided the original author and source are credited.

\section{INTRODUCTION}

$\mathrm{D}$ eep drawing is a manufacturing operation in which a thin sheet metal is deformed plastically to take the form of the punch or the die geometry. Regarding its advantages, it is being used increasingly especially within the aerospace, aircraft and automotive industries [1-2]. Each final product requires appropriate tools for being formed in deep drawing.

Classical forming processes have serious difficulties to fulfill the requirements of cost reduction, lightweight materials, low energy consumption and improved parts quality [2]. Therefore, new manufacturing technologies have been developed and improved. One of the developed technologies and the most important formative processes is hydromechanical deep drawing (HDD). In this process, liquid is used as a pressurized medium to facilitate the deformation of a sheet (blank). 
There are many advantages for this process, over conventional metal forming processes, such as better surface quality, increased drawing ratio, forming of complex shapes, cost-effective parts and lower tool costs [2].

HDD process, illustrated in Fig. 1(b), is one type of sheet hydroforming. Comparing the HDD process with conventional deep drawing process (CDD) (Fig. 1(a)) it can be noticed some differences in tools, contact conditions and producing technology. The female die in the CDD process is replaced by a cavity filled with a fluid in the HDD process. The final form of the part is determined by the punch. In this process, when the punch penetrates in the die cavity, the liquid is pressurized and pushes the sheet onto the punch surface (Fig. 2). The fluid pressure in the cavity can be controlled by a valve. The use of the fluid makes possible to reduce the friction and to prevent metal-to-metal contact at the blank-die interface which improve the possibilities of obtaining a better geometry of the final products [3-6]. Warm pressurized fluid can be used to increase the formability of lightweight alloys $[7,8]$.

The study of the HDD process and the effects of the fluid pressure were the subject of several research works. Singh and Kumar [9] studied the HDD process to show the influence of the fluid pressure in the cavity on the thickness distribution and the surface quality of the final products. They found that thinning of the sheet at the corner zone which exists in traditional sheet forming processes was reduced in the HDD process. They also noticed that the thickness distribution became more uniform with the increase in the maximum fluid pressure in the cavity. Modanloo et al. [10] studied the effects of forming media on the HDD process experimentally and by simulation using a typical pressure path. Water and oil were used as a forming media. They reported that by using oil, thinning decreases at the punch corner radius. In another work [11] they investigated, by experiments and simulation, the effects of forming parameters (fluid pressure, punch velocity, friction coefficients, punch and die corner radius...) on the thinning ratio of the deformed part. They found that a higher corner radius of the punch and the die lead to an increase in thickness reduction. Moreover, an optimization method was presented to improve thinning ratio.

In order to improve formability of the parts in HDD process, Salahshoor et al. [12] investigated the effects of geometrical and process parameters on thickness distribution of cylindrical parts. The results showed that the pressure path has a great influence on the formability. By increasing the maximum fluid pressure, a reduction in thinning tendency is observed. Furthermore, by decreasing the friction in the blank-blank holder contact region, a decrease in thinning and maximum punch force was observed.

The effect of the initial pressurization during the HDD process was studied by Lang et al. [13]. The obtained results showed that the use of an initial pressurization has a significant influence on the first stages of forming. The rise of the pressure increases the surface of contact between the part and the punch surface which avoid the rupture in the contact area. However, for excessive values of the pressure, the rupture will take place in the critical zones of the part. In another work [14], they studied the influence of the fluid pressure in the cavity on the deformation of the part. In this work, a uniform pressure was applied on the lower surface of the sheet. They found that a high pressure in the cavity can reduce the thickness of the formed part. Hama et al. [5] established an experiment on the HDD process. The fluid pressure was measured in various positions (in the cavity, in the die corner and between the sheet and the die). From this experiment study, they showed that the drawing ratio in the HDD process is better compared to CDD process.

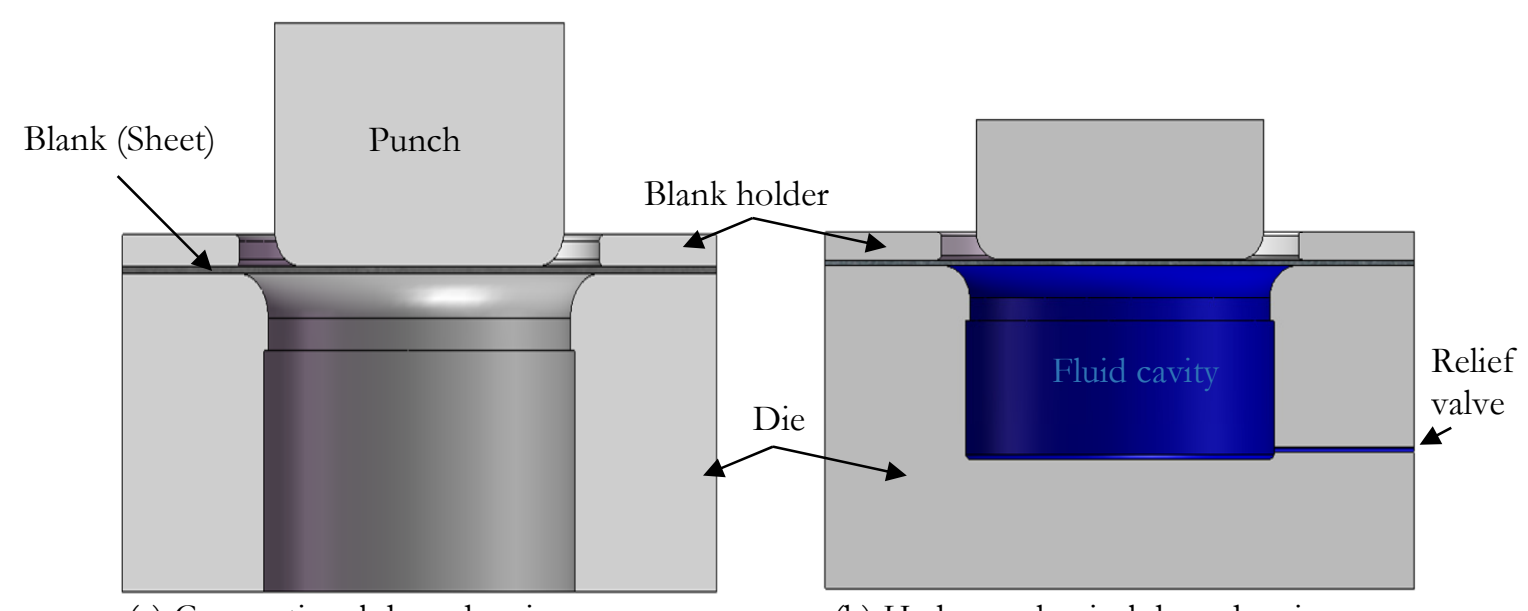

(a) Conventional deep drawing

(b) Hydromechanical deep drawing

Figure 1: Conventional and hydromechanical deep drawing processes. 
The previous work on modeling and simulating the HDD process of cylindrical parts by the authors [15] has established the reliability of the proposed approach in the modeling of process parameters. An analytical model was proposed to consider the non-uniform pressure distribution in the cavity and for the hydrodynamic pressure of the fluid film in the blank-die contact region. Based on this previous work, this study focuses on the comparison of conventional and hydromechanical deep drawing processes by performing a finite element analysis. The fluid cavity pressure effect on the plastic strain and the thickness distribution has been investigated.

\section{NUMERICAL MODELING}

\section{$\mathrm{F}$}

Finite element model inite elements simulations of conventional and HDD of a cylindrical cup are performed using the ABAQUS/Explicit software. In the numerical modeling, the two processes (CDD and HDD) are involved at the same initial conditions: the same geometry of the instrument, the same boundary conditions, the same applied loads, etc.

The deep drawing process involves a blank and an instrument consisting of three main parts: blank holder, punch and die (Fig. 1 and Fig. 2). In this case, considering the cylindrical symmetry, only one half of the blank and the instrument are analyzed in 2D. In the meshing of the blank the following initial conditions are used: continuum media; axial symmetry; and four node elements (CAX4R). During the process, the punch, the blank holder and the die are considered as rigid, while the blank is deformable. The die is constrained fully and the punch can move only along the vertical direction. The punch motion is prescribed with a constant velocity. A constant blank holder force throughout the process is applied.

Uniform friction conditions are assumed for all contacting surfaces. The Coulomb friction model is used to describe the contact between the blank and the tooling surfaces. The friction coefficients are adopted based on previous works in this field [17-19]. Tab. 1 summarizes the friction coefficient values for different contacts.

\begin{tabular}{ccc}
\hline Contact zone & HDD & Friction coefficient \\
Blank-Die & 0.05 (Presence of fluid) & 0.08 (Boundary contact) \\
Blank-Punch & 0.1 (Dry contact) & 0.1 (Dry contact) \\
Blank-Blank holder & 0.08 (Boundary contact) & 0.08 (Boundary contact) \\
\hline
\end{tabular}

Table 1: Friction coefficient values.

The fluid pressure variation in the die cavity is one of the most important parameters in the HDD process, because wrinkles appear if the pressure is not sufficiently high. In contrast, the blank is damaged by fracture if the pressure is too high [4]. During the simulation of this process, the modeling of the fluid-structure interaction is the principal difficulty. In a previous work [15], the authors give a detailed study of this problem. The fluid was represented by a non uniform pressure load applied on the lower surface of the blank. An analytical model was given to define the variation of the pressure in the cavity and the distribution of the pressure of the fluid between the blank and the die, this analytical model was implemented into the ABAQUS/Explicit code using the user defined subroutine VDLOAD. This developed program is used in the present work to take into account the fluid pressure in the cavity and the hydrodynamic pressure of the fluid film between the blank and the die.

\section{Material parameters}

The blank thickness is $1.12 \mathrm{~mm}$. The blank is made of an aluminum alloy (AA5086). Elasto-plastic material behavior with isotropic hardening is supposed. Hooke's model with a Young modulus E $=67293 \mathrm{MPa}$ and a Poisson ratio $\nu=0.3$ is used to describe the elasticity. The Von Mises yield criteria is used for the plasticity to describe the isotropic characteristics of the blank material. The hardening behavior is described by following relation:

$$
\bar{\sigma}=\sigma_{0}+k(\bar{\varepsilon})^{n}
$$


(a)

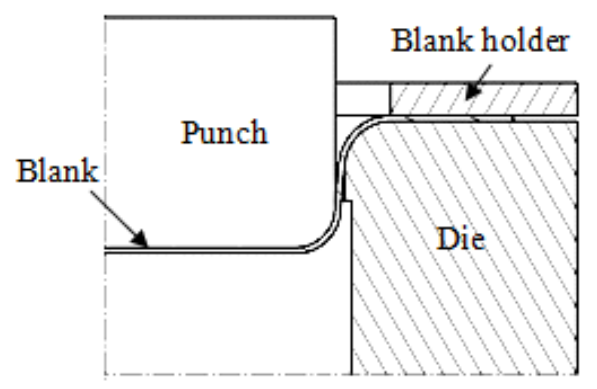

(b)

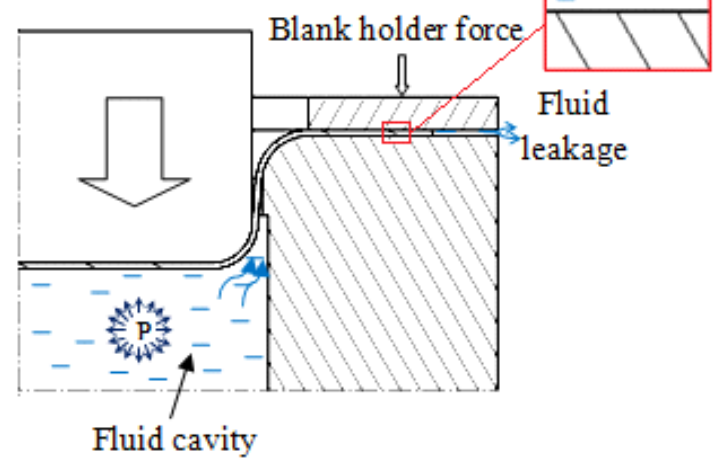

Figure 2: Schematic representation of: (a) conventional deep drawing process and (b) hydromechanical deep drawing process principle.

where $\bar{\sigma}$ and $\bar{\varepsilon}$ are the equivalent stress and the equivalent plastic strain, respectively, $\sigma_{0}=143.2 \mathrm{MPa}$ is the yield stress, $K$ $=296.9 \mathrm{MPa}$ is the hardening coefficient and $n=0.40$ is the strain hardening exponent. These material parameters are obtained from a bi-axial tensile test [16].

During the deep drawing, the blank is subjected to high plastic deformations. Sometimes these deformations become excessive, so that necking begins to appear in critical regions of the part. This represents the limit of the formability and the beginning of the fracture. The term formability is generally used to describe the ability of a material to be plastically deformed without necking or fracture. To characterize a material formability, forming limit diagrams (FLD) are widely used [20]. FLD is a plot of the combination of the minor and major strains at the beginning of localized necking. It becomes an essential tool in metal forming industry and also during finite element analysis or forming simulation. In this work, in order to evaluate the severity and the feasibility of the forming operation for the two processes, the FLD of the AA5086 aluminum alloy is used. This FLD is obtained by a biaxial tensile test [16].

\section{RESULTS AND DISCUSSION}

$\mathrm{F}$ ig. 3 shows the equivalent plastic strain distribution, for the two processes, after $15 \mathrm{~mm}$ of punch displacement. In CDD process, maximum plastic strain value of approximately $38 \%$ is observed. This value decreases to $25 \%$ in the HDD process. For the two processes, the blank undergoes maximum deformation on punch and die corners. In the HDD process, a strain concentration occurred at the die corner as a result of bending. For the same reason and because of excessive stretching, high strain concentration is present at the punch corner in the CDD process. This can be clearly observed in Fig. 4 where the evolution of the plastic strain in the radial direction for a punch displacement of 15 $\mathrm{mm}$ is shown. We can see that the use of the fluid in the HDD process has a significant influence on the strain distribution. Maximum strain values are observed at the punch and the die corner where the blank is exposed to bending. Comparing the obtained results for the strain distribution, a large difference can be seen at the punch corner, at the die corner and at the blank-punch contact region. By contrast, a small difference is observed at the blank-blank holder contact zone (radial position is upper than $40 \mathrm{~mm}$ ). The force exerted by the blank holder on the blank at this region supplies a restraining force which controls the metal flow and influences the strain distribution. In the HDD process, in addition to the blank holder force, there is high fluid pressure under the blank. The force generated by this pressure, which is opposite to the blank holder force, has also an influence on the strain distribution. This can explain the observed reverse behavior of the strain at this zone. According to the numerical results in Fig. 3 and Fig. 4, the resulting plastic strain distribution is more uniform and the maximum strain values are lower in the HDD process compared to CDD process. In CDD process, by the displacement of the punch, a radial traction force on the blank is produced. The absence of a fluid lubricating in the zone of contact blank-die causes a strong friction force in particular on the die corner. This implies a reduction in the slip of the blank under the blank holder. In this case, the force applied by the punch draws the blank and a plastic deformation dependent on this force is produced. Consequently, a radial effort is created on the blank, thus a higher traction force is necessary for the deformation. Drawing becomes significant producing an excessive thinning of the blank on the punch corner. As the traction force that the bank can resist is limited by the tensile strength of the used 
material, the fracture can appear with the advancement of the punch. It is possible to prevent the strain concentrations by using suitable process parameters such as a good lubrication and an appropriate blank holder force.

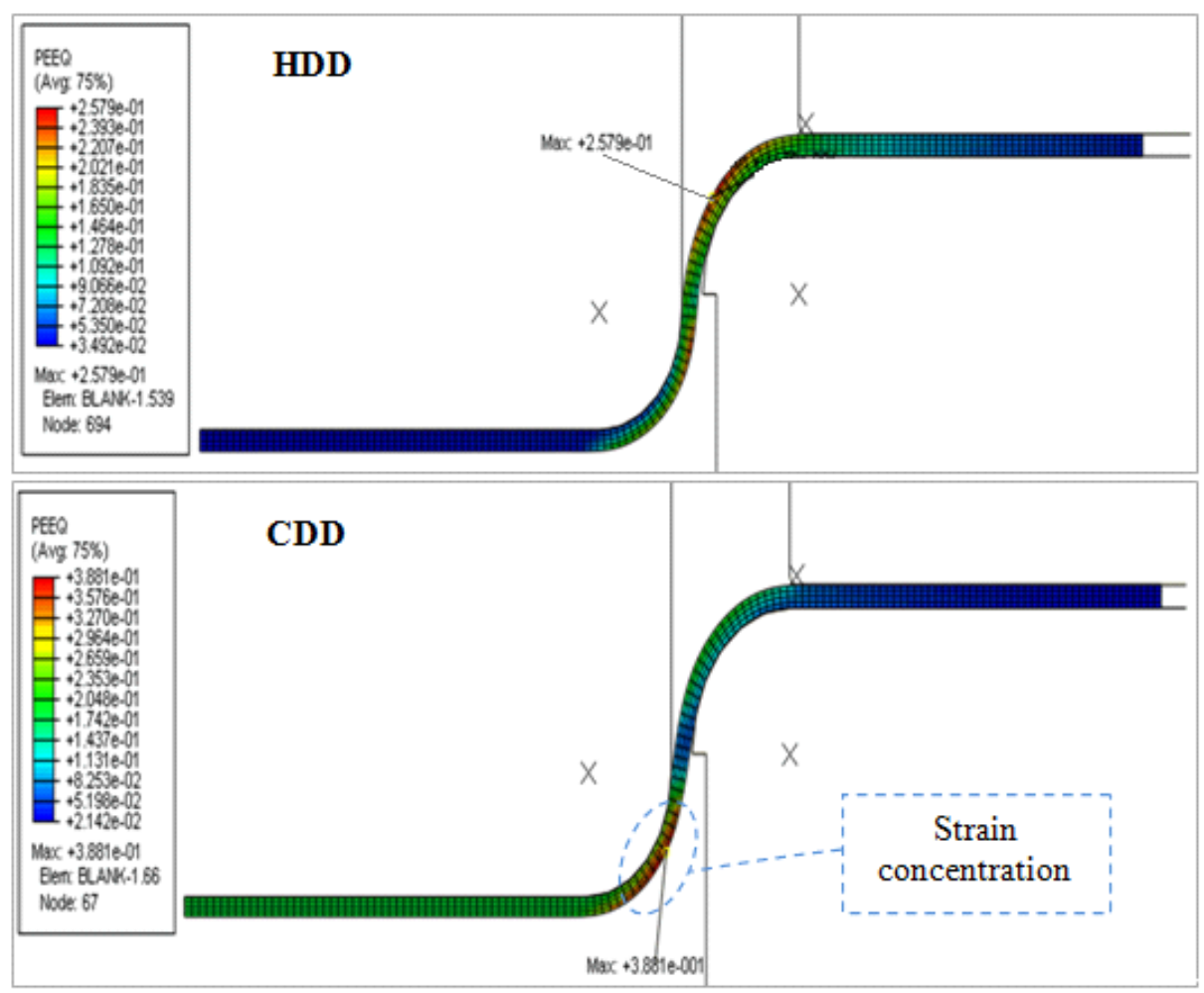

Figure 3: Equivalent plastic strain distribution after $15 \mathrm{~mm}$ of punch displacement.

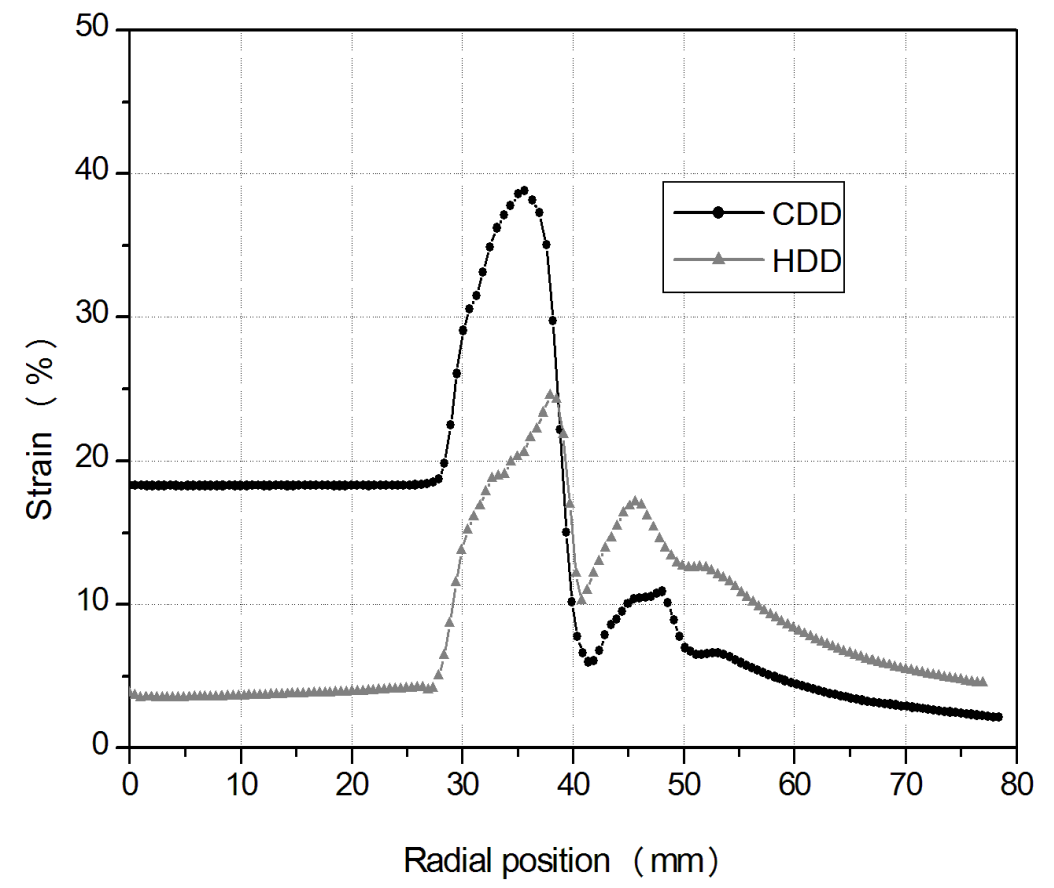

Figure 4: Strain distribution in the radial position after $15 \mathrm{~mm}$ of punch displacement. 
An evaluation of the forming severity for the two processes is obtained by comparing the numerical results for the strain distributions with the experimental FLD of the used material. The FLD is plotted in a principal strain reference. Major strain which is the largest in algebraic value is on the ordinate. Minor strain which is the smallest in algebraic value is on the abscissa. Although the major strain is always positive because it is obtained by stretching, the minor strain is may be either positive in the case of stretching or negative in shrink drawing case. The upper region of the FLD represents necking and fracture. The safe region in which no fracture is expected is the region under the FLD. Numerical major and minor strains distribution and experimental FLD of the used blank material are presented in Fig. 5. For the two processes, the material exhibits slightly different principal strains and the produced parts have not the same formability. The results prove that, until $15 \mathrm{~mm}$ of punch displacement, the parts can be formed without fracture. For CDD process, beyond this punch displacement (Fig. 6), excessive thinning followed by a fracture can occur on the punch corner where the most dangerous points are located. From these results, it can be concluded that the use of the fluid pressure gives better formability and drawing ration. According to Sadegh-yazdi et al. [21], formability can be improved using an optimum fluid pressure path for radial and cavity pressures in HDD process.

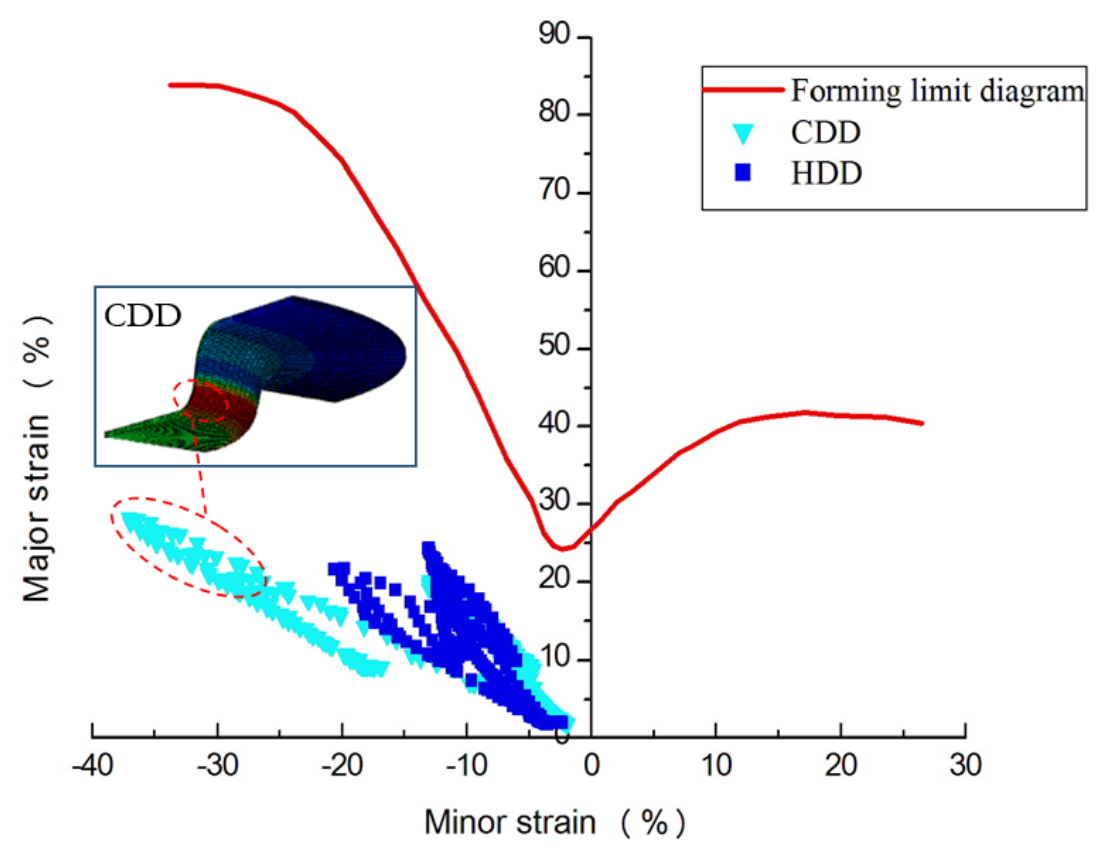

Figure 5: Experimental FLD and numerical results for principal strains distribution after $15 \mathrm{~mm}$ of punch displacement.

The quality of the formed part not only depends on the formability but also depends on other factors such as thinning. To study the influence of the fluid pressure on the blank thickness distribution, a comparison of the blank thicknesses for the two processes is carried out and the result is shown in Fig. 7. It can be shown that the thickness distribution is significantly influenced by the presence or not of the fluid in the cavity. As shown, there is a tendency of thinning at the punch corner in the CDD process with a minimum thickness of $0.81 \mathrm{~mm}$. When compared with CDD process, less thinning is observed in HDD process. The decrease in thinning tendency in the HDD process is due to the presence of the pressurized fluid, as it acts as an effective lubrication which facilitates the flow of the blank. And also, the force generated by the fluid pressure in the cavity acts as uniform stress during the forming operation. As reported in the literature [22], the use of the pressure force, in the HDD process, reduces product defects like thinning. More uniformity of thickness distribution can be obtained by using the initial bulging of the blank [23].

\section{CONCLUSION}

$\mathrm{I}$ $\mathrm{n}$ this paper, The HDD process and CDD process have been simulated by means of the FE software package ABAQUS/Explicit. The plastic deformation distribution and the formability of the formed part were discussed. The obtained results indicate that the fluid pressure under the blank plays an important role in the lubrication effect, the 
plastic deformation and the thickness distributions.

Comparing the obtained results for the strain distribution, a large difference can be seen at the bottom and the corner of the punch. In HDD process, the plastic strain distribution is more uniform compared to CDD process and the maximum strain values are lower.

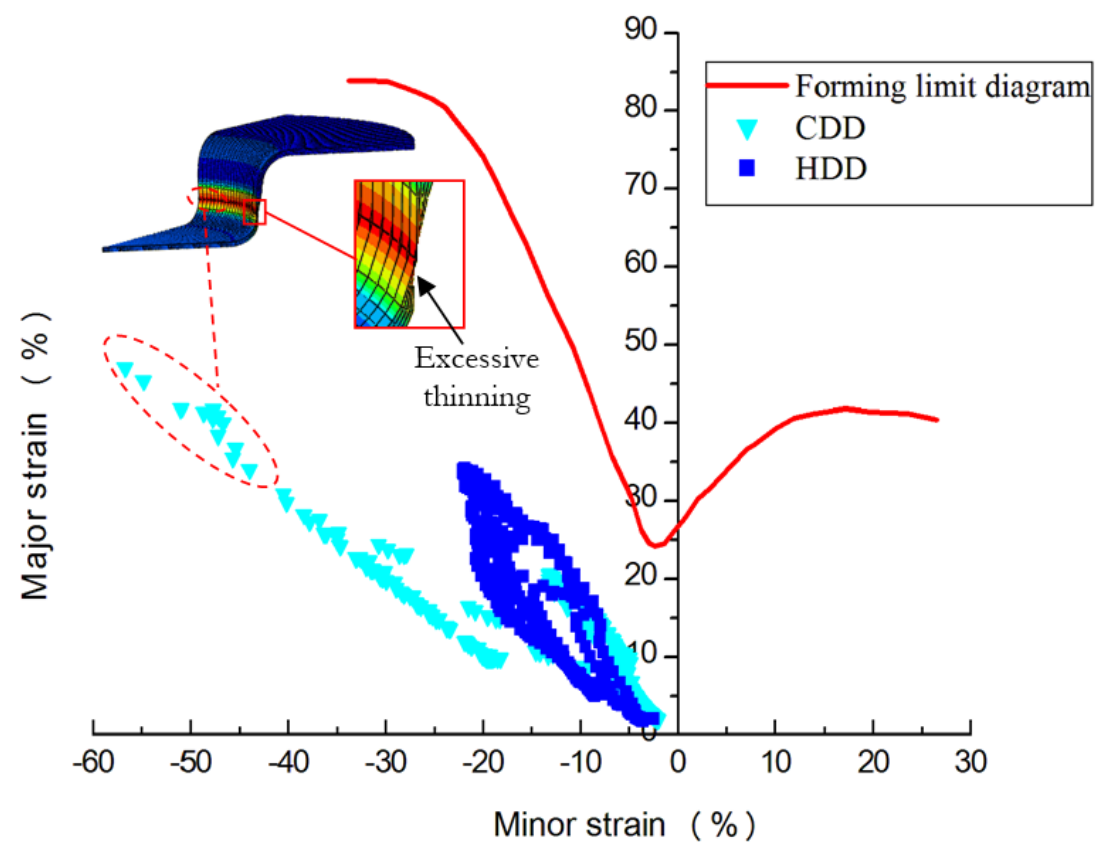

Figure 6: Experimental FLD and numerical results for principal strains distribution after $20 \mathrm{~mm}$ of punch displacement.

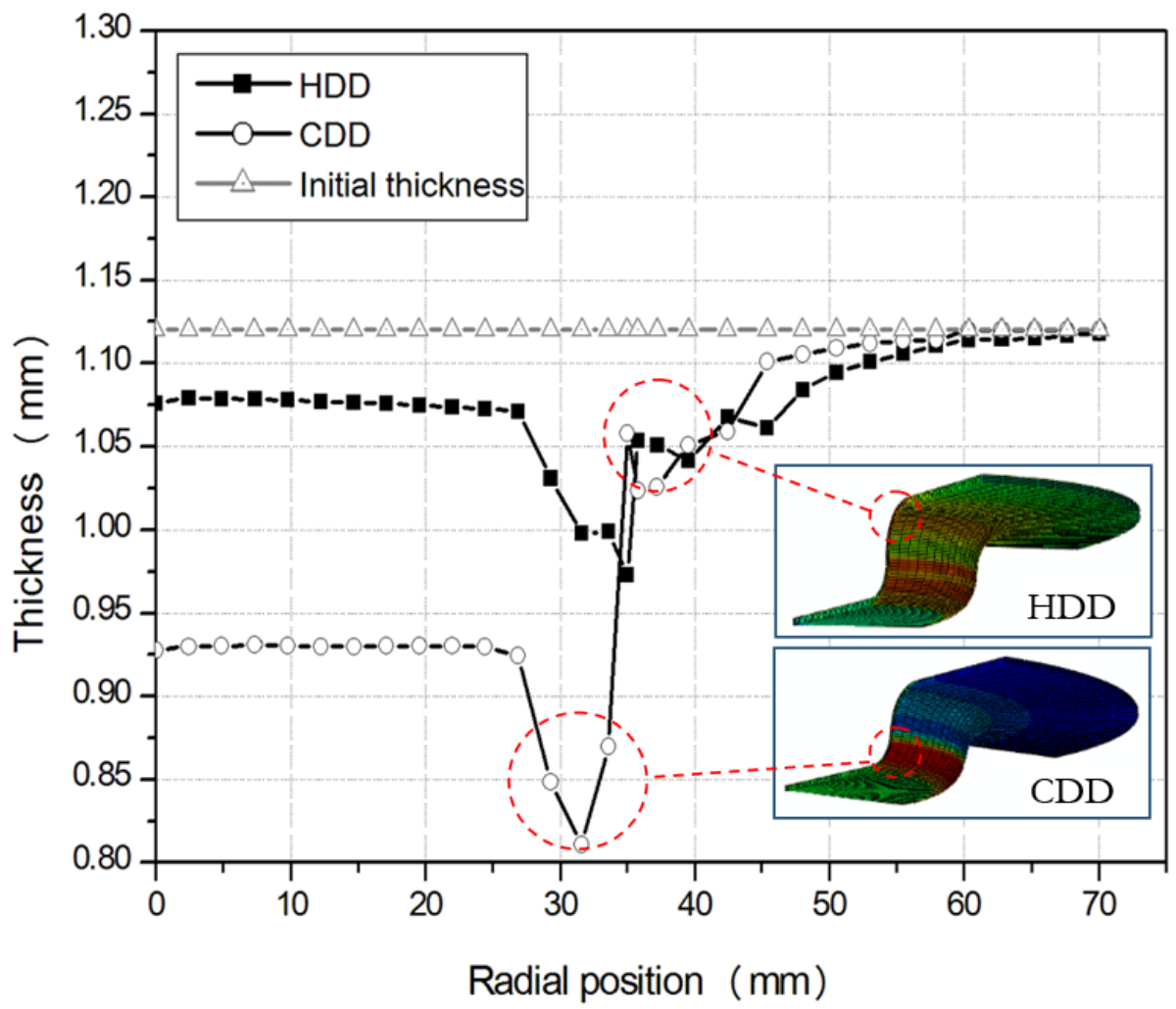

Figure 7: Thickness distribution in the radial direction after $15 \mathrm{~mm}$ of punch displacement. 


\section{REFERENCES}

[1] Singh, H. (2003). Fundamentals of Hydroforming, Society of Manufacturing Engineers (SME), USA.

[2] Koc, M. (2008). Hydroforming for advanced manufacturing, CRC Press, USA.

[3] Hama, T., Kurisu, K., Matsushima, K., Fujimoto, H., Takuda, H. (2009). Outflow characteristics of a pressure medium during sheet hydroforming, ISIJ International, 49 (2), pp. 239-246. DOI: 10.2355/isijinternational.49.239.

[4] Zhang, S.H., Danckert, J. (1998). Development of hydro-mechanical deep drawing, Journal of Materials Processing Technology 83, pp. 14-25. DOI: 10.1016/s0924-0136(98)00039-9.

[5] Hama, T., Matsushima, K., Kitajima, T., Fujimoto, H., Takuda, H. (2009). Correlation between sheet deformation and hydraulic pressure variation during sheet hydroforming, ISIJ International, 49 (11), pp. 1736-1743. DOI: 10.2355 /isijinternational.49.1736.

[6] Alizad-Kamran, M., HoseinpourGollo, M., Hashemi, A., Hossein Seyedkashi, S.M. (2018). Determination of critical pressure in analyzing of rupture instability for hydromechanical deep drawing using advanced yield criterion. Archives of civil and mechanical engineering, 18, 103-113. DOI.org/10.1016/j.acme.2017.05.008.

[7] Gaoshen, C., Chuanyu, W., Dongxing, Z. (2018). Investigation on the Effect of Type of Cooling on the Properties of Aluminum Alloy during Warm/Hot Hydromechanical Deep Drawing. Symmetry, 10, 362. DOI:10.3390/sym10090362.

[8] Acara, D., Türközb, M., Gediklia, H., Selçuk, H. H., Necati, C. Ö. (2017). Warm Hydromechanical Deep Drawing of AA 5754-O and Optimization of Process Parameters. Journal of Engineering Materials and Technology, 140(1), DOI:10.1115/1.4037524.

[9] Singh, S. K., Ravi Kumar, D. (2008). Effect of process parameters on product surface finish and thickness variation in hydro-mechanical deep drawing, Journal of Materials Processing Technology, 204, pp. 169-178. DOI: $10.1016 /$ j.jmatprotec.2007.11.060.

[10] Modanloo, V., Gorji, A., BakhshiJooybari, M. (2016). Effects of forming media on hydrodynamic deep drawing. Journal of Mechanical Science and Technology, 30(5), 2237-2242. DOI 10.1007/s12206-016-0433-x.

[11] Modanloo, V., Gorji, A., BakhshiJooybari, M. (2018). A comprehensive thinning analysis for hydrodynamic deep drawing assisted by radial pressure. Iran J Sci Technol Trans Mech Eng, pp. 1-8. DOI: 10.1007/s40997-018-0221-6.

[12] Salahshoor, M., Gorji, H., Bakhshi-Jooybari, M. (2019). Analysis of the effects of tool and process parameters in hydrodynamic deep drawing assisted by radial pressure. J Braz Soc Mech Sci Eng, 41, 145. DOI: $10.1007 /$ s40430-019-1648-4.

[13] Lang, L., Danckert, J., Nielsen, K. B. (2004). Investigation into the effect of pre-bulging during hydromechanical deep drawing with uniform pressure onto the blank, International Journal of Machine Tools and Manufacture, 44, pp. 649-657. DOI: 10.1016/j.ijmachtools.2003.11.004.

[14] Lang, L., Danckert, J., Nielsen, K. B. (2004). Study on hydromechanical deep drawing with uniform pressure onto the blank, International Journal of Machine Tools and Manufacture, 44, pp. 495-502. DOI: $10.1016 /$ j.ijmachtools.2003.10.028.

[15] Abbadeni, M., Zidane, I., Zahloul, H., Fatu, A., Hajjam, M. (2017). Finite element analysis of fluid-structure interaction in the hydromechanical deep drawing process, Journal of Mechanical Science and Technology 31 (11), pp. 5485-5491. DOI: 10.1007/s12206-017-1043-y.

[16] Zidane, I. (2009). Développement d'un banc d'essai de traction biaxiale pour la caractérisation de la formabilité et du comportement élastoplastique de tôles métalliques, thèse de doctorat, INSA Rennes.

[17] Zhang, SH., Jensen, MR., Nielsen, KB., Danckert, J., Lang, LH., Kang, DC. (2003). Effect of anisotropy and prebulging on hydromechanical deep drawing of mild steel cups. J Mater Proc Technol, 142, pp. 544-50. DOI: $10.1016 / \mathrm{s} 0924-0136(03) 00656-3$.

[18] Fazli, A., Dariani, BM. (2006). Theoretical and experimental analysis of the axisymmetric hydromechanical deep drawing process. Proc Inst Mech Eng B: J Eng Manuf, 220, pp. 1429-1437. DOI: 10.1243/09544054JEM472.

[19] Schuler GmbH, (1998). Metal forming handbook, Springer-Verlag Berlin Heidelberg.

[20] Giuliano, G., Bellini C., Sorrentino, L., Turchetta, S. (2018). Forming process analysis of an AA6060 aluminum vessel. Frattura ed Integrità Strutturale, 45, pp. 164-172. DOI: 10.3221/IGF-ESIS.45.14.

[21] Sadegh-yazdi, M., Bakhshi-Jooybari, M., Shakeri, M., Gorji, H., Khademi, M. (2018). Optimization of pressure paths in hydrodynamic deep drawing assisted by radial pressure with inward flowing liquid using a hybrid method. Int $\mathrm{J}$ Adv Manuf Technol, 97, 2587-2601. DOI.org/10.1007/s00170-018-2023-9. 
[22] Atul, T. S., Lenin Babu, M. C. (2018). A review on effect of thinning, wrinkling and spring-back on deep drawing process. Proc IMechE Part B: J Engineering Manufacture, 233:4, pp. 1011-1036. DOI: 10.1177/0954405417752509.

[23] Desu, R. K., Singh, S. K., Gupta, A. K. (2016). Comparative study of warm and hydromechanical deep drawing for low-carbon steel. Int J Adv Manuf Techno, 85, pp. 661-672. DOI 10.1007/s00170-015-7819-2. 